\title{
Combination and Application of Data Mining in Knowledge Management of Colleges and Universities
}

\author{
Liu Xueping \\ Engineering Training Center \\ Shenyang Aerospace University \\ Shenyang City, China
}

\begin{abstract}
Nowadays, with the development of the society, knowledge management has already become an important means of management to upgrade the level of discipline construction in colleges and universities, improve students' satisfaction and enhance colleges and universities' comprehensive competitiveness. AS an advanced means of information technology at present, data mining plays a rather important role in promoting knowledge management in colleges and universities. This paper mainly analyzes the combination of knowledge management and data mining in colleges and universities, and specifically describes the important role of data mining in colleges and universities' knowledge management.
\end{abstract}

Keywords-Data mining; Knowledge management; Combination; Management system establishment; Information technology

\section{INTRODUCTION}

Institutions of higher learning are a kind of knowledge intensive organizations with a huge amount of knowledge capital and also bear the important task to disseminate, create, use and optimize knowledge. As colleges and universities have a huge amount of high-level knowledgeable talents and information resources, and enjoying exceptional advantages in relevant policy and scientific research environment, it is rather feasible to conduct knowledge management in colleges and universities. Therefore, knowledge management has already become an important task in the reform of colleges and universities while being accepted by the majority of colleges and universities with the passing of time. Knowledge management is the management of information and persons. Through knowledge management, we can delicately combine information processing and the innovation ability of people so as to promote the organization to have stronger comprehensive competence. With data mining, an acquired information technology, more potential information can be obtained after processing and analyzing data. Data mining has provided powerful support for knowledge management and plays an important promotional role in improving the efficiency of knowledge management. Meanwhile, it is also conducive to the sharing and acquisition of knowledge.

\section{CURRENT StATUS OF DOMESTIC AND ForEIGN}

\section{RESEARCHES}

It can be found from searching the CNKI (China National Knowledge Infrastructure) that tens of thousands of literatures can be obtained through retrieving the keyword knowledge management, and most of the, were created in recent ten years. Therefore, we can see that research into knowledge management reached its new climax ten years ago. More than two hundred literatures can be searched out by searching highly effective knowledge management. Therefore, we can see that there are still few dedicated literatures researching knowledge management in colleges. During the process of searching both data mining and knowledge management, we can find that that only less than ten relevant literatures.

Through retrieving, we have found about double times foreign literatures about knowledge management and data mining of domestic literatures with superior width and breadth.

\section{SPECIFIC REQUIREMENTS TO SOFTWARE DURING THE}

\section{PROCESS OF INTEGRATING DATA MINING AND}

\section{KNOWLEDGE MANAGEMENT}

The integration and application of data mining and knowledge management have also proposed higher requirements to a series of relevant equipment and deeper specific requirements to the server, computer hardware, knowledge management platform and utility software.

\section{A. Specific Requirements to Knowledge Management \\ Platform}

In order to conduct knowledge management in colleges and universities, a perfect college knowledge management platform must be established because it can provide visual operation interface. Basic functions needed include: tool interface, data interface, internal or external data mining, knowledge database, information retrieval, knowledge construction, evaluation and management and organization communication.

A knowledge management platform can be divided into the service level, demonstration level, storage level and processing level. The so-called demonstration level is a visual interface to all the users and is mainly used for receiving customers' tasks and interpersonal interaction. 
The so-called service level refers to process, schedule and implement tasks and give feedbacks while the processing level is mainly used for pre-treatment of data, data mining and knowledge discovery. The storage level mainly includes knowledge base and database of colleges and universities.

According to the specific characteristics of the institutions of higher learning, the knowledge management platform needs to be able to achieve the basic functions of the basic functions of the following several notable features. First, the requirements for the quality of the interface need to be improved. As a result of higher learning institutions is a collection of administrative, social services and scientific research and other related tasks in a complex organization, therefore, the need to deal with the amount of data is also very large, so the interface quality must meet the relevant requirements, with the ability to handle complex tasks, so as to protect data processing depth and efficiency. Secondly, for the built-in data mining capabilities, the higher institutions because of the data covered by the very rich and wide range of data, so that the data is small, relatively simple structure of the data can be used to handle data mining functions, skills, save time, and can effectively use the resources within the organization, can be described as multiple. Third, communication function must also be promoted, the University as a knowledge intensive organization, therefore, function and discipline has a lot of poor, so it is only sharing and Knowledge Innovation exchange function is particularly important. Fourth, the incentive system should be relatively perfect. As the incentive system is not only reflected in the evaluation function of the platform, but also can be reflected in the management, through the improvement of management staff can effectively improve the process of knowledge management.

\section{B. Specific Requirements to Data Mining Tools}

The knowledge covered by institutions of higher learning can be divided into two main parts. First, it is to manage knowledge. It is also the teaching of colleges and universities, and logistics and other related departments for the management to learn related knowledge. Second, when scientific research. It is also the subject of research and teaching of the relevant professional knowledge. Management knowledge is mainly related to the management personnel and administrative personnel in Colleges and universities, and the research is also related to the professors and researchers of higher learning institutions. Because of the different users, the data mining and knowledge management are compatible with the application of data mining software.

Most of the data required for the management and administration of the institutions of higher learning are recorded in the information system of universities and colleges. For example: computer information, campus card consumption records, etc., so the data volume is large, and the range is very wide, but with the specific characteristics of the structure, so that the application of such data mining tools can be built in the knowledge management platform, which is conducive to data storage and access, can effectively improve and improve mining data. The main functions can be divided into three parts, the classification, the prediction and the evaluation. The main method can choose the different methods, such as trend extrapolation, regression analysis, hierarchical gap and feature analysis. The results of the mining should also have a very strong visibility, it is best to provide users with intuitive graphics, is conducive to the user's understanding.

For the scientific research knowledge in Colleges and universities, it is mainly related to the teaching and research scholars. The main results are as well as the experimental data and the subjective speculation. For mining low data, it can also use the interior of the mining tools. However, for the relatively high data mining requirements, you need to use professional data mining software for data mining, which is connected to the knowledge management platform of colleges and universities.

\section{StRategy to InTEgrate Data Mining AND}

\section{KNOWLEDGE MANAGEMENT}

For knowledge management, the basic functions of the knowledge management include internal, external, intermediary and from the four specific branches, the first three specific branches of information technology has a strong dependence, can be used to implement data mining. The process of the specific conditions of the matching and selection, activation and application of these parts, that is, after the data preprocessing, the need to filter out the relevant data, according to the requirements of the corresponding data mining, mining results, and finally the record in practice. Specific strategies to combine knowledge management and data mining include:

\section{A. Establish Sound Auxiliary Knowledge Management}

\section{System}

The essence of knowledge management is periodical management. In order to improve the comprehensive competition ability, and knowledge management system is a comprehensive and systematic engineering. Data mining for the construction of knowledge management system and its auxiliary functions reflect the in the use of knowledge is in charge of the management of colleges and universities to carry on the management and decision-making, knowledge management department of information of collection and processing.

\section{B. Externalization of Knowledge}

The so-called externalization of knowledge refers to the process of obtaining knowledge related to the organization from outside it, collate and discover internal knowledge of an organization and store this knowledge. The key to complete externalization of knowledge lies in several specific steps: discovering knowledge, collating knowledge and processing invisible knowledge, etc.

There are two premises here: first, the discovery of knowledge can not only depend on the information technology, but also need to dig the results of the subjective staff training, explain their reasoning to complete the semantic knowledge; second, you need to dig the object and transform into logical data. Using data mining technology, knowledge discovery has several methods: knowledge discovery and abnormal value. If there is a rule that can be used to determine the mining 
area, and if there is a certain rule, using fuzzy technology, statistical methods can be obtained from the University's decision analysis, determine the validity of the program, and draw patterns similar to the decision process.

\section{Internalization and Mediation of Knowledge}

The so-called internalization of knowledge is to find specific personnel's demands for knowledge and provide support for them with knowledge. The main factor of knowledge internalization lies in classifying and summarizing knowledge and fully exploring personnel's interests. The so-called knowledge medium refers to some knowledge without codes in an organization. For this kind of knowledge, relevant specific means can be taken to match knowledge demanders and knowledge to provide a channel for communication between the two. In knowledge internalization and medium function, data mining mainly services to data specific characteristics of personnel in colleges and universities.

\section{Promote Management of Library Management in Colleges and Universities with Data Mining}

Libraries in colleges and universities are a special resource in organizing institutions of higher education as they provide a huge amount of knowledge like an entity warehouse of knowledge. Effective management of knowledge in libraries can provide great help to knowledge management in colleges and universities. At present, many Chinese library experts have already begun to apply data mining in library management. Data mining of libraries include three aspects: book information, lending information and readers' information. Synthesizing data mining of the three specific objects can effectively provide powerful support for libraries in constructing resources and providing personalized services and reader services to improve the quality of libraries' service and provide more personalized services for different readers.

\section{E. Give Play to the Management Function}

Knowledge management is a systematic project, mainly including several aspects, such as platform development, cultural training and system construction, and involving a lot of data operation in practice. Therefore, it can only have technical support in practice by relying on data mining to effectively facilitate users and improve the efficiency of knowledge management. Combination of knowledge management and data mining in colleges and universities can effectively shorten the cycle of knowledge management, thus it is of positive practical significance for college and university managers. In practice, the two can influence each other so as to improve the comprehensive accomplishment of users. Quality of data mining operators could directly affect the quality of data mining, and knowledge management could also improve data mining personnel's recognition of knowledge so as to improve the quality of data mining and also make knowledge become more independent, clear and acceptable.

\section{CONCLUSION}

To sum up, at present, with the progress of science and technology, many relevant scholars, both domestic and foreign, begin to study combining data mining and knowledge management, which is gradually being widely used in colleges and universities. The effective combination of the two improves the work efficiency of colleges and universities. However, at present, colleges and universities in China still lack operation staffs with relevant professional accomplishment. Therefore, data mining cannot comprehensively support knowledge management to a great extent, but it is believed that over time, when the problems are gradually solved, the combination of knowledge management and data mining in colleges and universities will surely bring greater support to the healthy and stable sustainable development of colleges and universities.

\section{REFERENCES}

[1] Combination and Application of Data Mining in Colleges and Universities' Knowledge Management, Guo Shaoqin, Scientific and Technological Information of China, 2014.

[2] Research into Integration and Application of Data Mining and Knowledge Management, We Chengwei, Jiang Dongmin, Management Science and Engineering Management in China, 2009.

[3] Application of Data Mining Technology in Information Construction, Quan Yun, China Science and Technology Expo, 2015.

[4] Research and Realization of Knowledge Management System based on Data Mining, Zhou Zhiming, Shandong Medical College, 2008.

[5] Data Mining and Knowledge Management of Thematic Database Take "Database of Coal Mine Accidents in China" as an Example. Du Pingping, Li Ming - Chinese Academy of Science, 2005.

[6] Text Mining, Data Mining and Knowledge Management, Han Kesong, Wang Yongcheng, Journal of Intelligence, 2015.

[7] Knowledge Management and Data Mining, Yang Chao, China Science and Technology Expo, 2010.

[8] Discussion of Data Mining Technology in Knowledge Management, Liu Ruyuan, Liu Wenxue, Chen Shan, Business Herald, 2015.

[9] Research of the Application of Data Mining in Enterprises' Knowledge Management, Xie Haiying, Enterprises' Science and Technology and Development, 2011.

[10] Research into the Application of Data Mining in Client Knowledge Management, Yang Weina, Heibei Enterprises, 2013. 